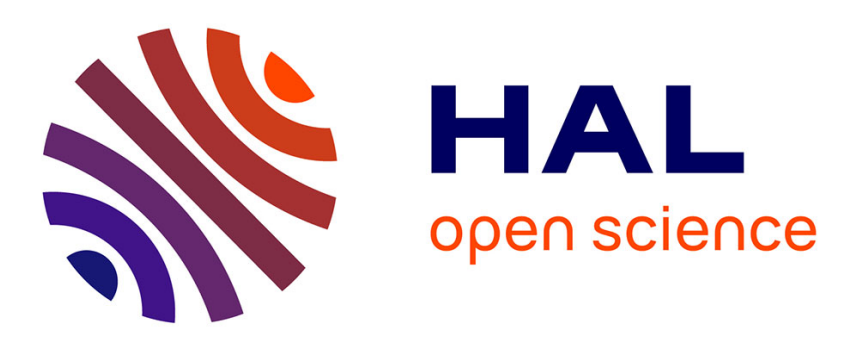

\title{
Le lieu en histoire des sciences. Hypothèses pour une approche spatiale du savoir géographique au XVIe siècle Jean-Marc Besse
}

\section{To cite this version:}

Jean-Marc Besse. Le lieu en histoire des sciences. Hypothèses pour une approche spatiale du savoir géographique au XVIe siècle. Mélanges de l'École française de Rome - Italie et Méditerranée, 2004, 116 (2), pp.401-422. halshs-00113236

\section{HAL Id: halshs-00113236 \\ https://shs.hal.science/halshs-00113236}

Submitted on 11 Nov 2006

HAL is a multi-disciplinary open access archive for the deposit and dissemination of scientific research documents, whether they are published or not. The documents may come from teaching and research institutions in France or abroad, or from public or private research centers.
L'archive ouverte pluridisciplinaire HAL, est destinée au dépôt et à la diffusion de documents scientifiques de niveau recherche, publiés ou non, émanant des établissements d'enseignement et de recherche français ou étrangers, des laboratoires publics ou privés. 
Tome 116 - 2004 - 2

p. 401-422

\section{Le lieu en histoire des sciences.}

Hypothèses pour une approche spatiale du savoir géographique au $\mathrm{XVI}^{\mathrm{e}}$ siècle $^{1}$

\section{Introduction}

La question du lieu n'est pas à proprement parler une nouveauté pour l'historien. Celui-ci entretient avec elle des relations anciennes, qui structurent de manière intime le mouvement même de sa recherche ainsi que sa pratique d'écriture. Comme l'indique Michel de Certeau, en effet, « le geste qui ramène les 'idées' à des lieux est précisément un geste d'historien » (M. de Certeau, 1975, p. 63). Un geste qui consiste à parcourir le chemin qui va du fait (quelle qu'en soit la nature) à ses conditions, et parmi ces conditions se trouve celle, fondamentale, du lieu de sa production, celle de son « où ? » et aussi celle de la nature propre de ce « où ? ». Il n'est pas insensé, en ce sens, d'envisager le prolongement, vers l'histoire des sciences, de cette opération historiographique, dont il s'agirait alors de définir la portée, les enjeux, et aussi la possible application à l'activité scientifique. Les savoirs scientifiques entretiennent eux aussi des relations complexes avec les «sites » où ils apparaissent : le 
« terrain », le « cabinet », le « laboratoire », la « bibliothèque », le « musée », la « salle de cours », peuvent être considérés comme autant de lieux spécifiques au sein desquels des jeux d'acteurs, de rationalités et de savoirs sont mis en œuvre de façon hétérogène (voir, par exemple, M. Beretta, 2002 ; M.-N. Bourguet, C. Licoppe, et H. Otto Sibum, 2002).

De fait, dans le domaine spécifique de l'histoire et de la sociologie des sciences, la perspective d'une localisation du savoir a déjà fourni quelques résultats significatifs, renouvelant parfois l'idée même que l'on peut se faire de la pratique scientifique. L'histoire des sciences a progressivement mis en évidence le rôle constitutif des institutions dans l'élaboration du savoir scientifique, en s'intéressant à la création des Académies, en éclairant les mécanismes de patronage, en soulignant de manière générale la présence active des réseaux et des sociabilités savantes. De son côté la sociologie du savoir scientifique contemporain, a cherché à établir de façon très directe $l^{\prime}$ « historicité locale » ${ }^{2}$ des pratiques, des procédés, mais aussi des idées et des données produites au sein des laboratoires scientifiques. On se trouve donc aujourd'hui devant un faisceau de travaux convergents, auxquels manque peut-être encore l'unité d'une problématique d'ensemble.

Curieusement, les géographes eux-mêmes, jusqu'à une date récente, n'ont pas vraiment pris en compte cette dimension spécifique dans les études historiques et épistémologiques consacrées à la géographie. Certes, l'histoire de la géographie a beaucoup travaillé du côté de ce qu'on pourrait appeler les traditions nationales, en particulier pour ce qui concerne le $\mathrm{XIX}^{\mathrm{e}}$ et le $\mathrm{XX}^{\mathrm{e}}$ siècles ${ }^{3}$. Mais les géographes ou les historiens de la géographie ne sont rarement allés plus loin, le plus souvent, que les orientations qui viennent d'être esquissées. On se trouve donc encore, sur ce point, comme le reconnaissait David Livingstone (D.N. Livingstone, 1995), devant un horizon programmatique. Ce programme commence aujourd'hui d'être mis en œuvre, à la fois sur le plan des réflexions théoriques et sur celui des études de cas (F. Driver, 2001 ; M.H. Edney, 1997). Les principales avancées 
proviennent en fait, d'une part, des historiens de la cartographie (dans l'héritage des travaux de Brian Harley ; cf. J.B. Harley, 2000), et d'autre part des travaux qui envisagent de manière générale la géographie en relation avec l'histoire naturelle (C.W.J. Withers, 1995 ; D. Outram, 1996). Dans ces travaux, soulignons-le, la géographie n'est plus seulement considérée comme l'objet d'un ensemble d'investigations historiques, elle devient elle-même une méthode de travail dans l'histoire des savoirs.

C'est dans le prolongement de cette perspective historiographique qu'on aimerait ici se placer. Voilà l'hypothèse : il serait possible de faire de la géographie, en tant que telle, une structure de compréhension de l'histoire des sciences, et de l'histoire de la géographie en particulier. Plus précisément dit, la réflexion géographique, dans la diversité de ses orientations de recherche, constitue une ressource conceptuelle et méthodologique pour l'histoire des sciences (y compris les « sciences géographiques »).

Cependant, de quelle géographie s'agit-il, et, surtout, quelle est la conséquence pour la conception du lieu devant être engagée dans l'histoire des sciences ? Car l'appel à la géographie, très vite, provoque l'apparition d'un doute, ou au moins d'une question sur la perspective « localisatrice » qu'on vient d'évoquer. Il ne suffit pas de dire en effet qu'il existe des lieux de la production des idées, et que le travail de l'historien consiste à effectuer le trajet qui va des idées à leur lieu. Il faut aussi s'accorder sur ce qu'on entend précisément par lieu, ainsi que sur le mode particulier de liaison qui s'établit entre lieu et idée (pour continuer sur ce même registre). Or la géographie, dont on a pu dire longtemps qu'elle était par vocation « science des lieux », se rapporte aujourd'hui avec beaucoup de précautions à cette notion. Elle a, en particulier, abandonné progressivement l'idée de l'unicité et de la singularité incomparable du lieu géographique, pour envisager celui-ci au sein de dialectiques plus complexes, auxquelles le lieu participe mais dont il n'est qu'un élément (J.-M. Besse, 1994). 
La géographie est passée d'une problématique du lieu à une problématique de l'espace, au sein de laquelle la notion de localisation a été profondément remaniée.

Mais, à vrai dire, le concept d'espace, tout comme celui de lieu n'est pas étranger à l'historien. L'idée selon laquelle l'histoire se développe dans des espaces multiples, caractérisés par des configurations, des polarités, des maillages et des discontinuités, l'idée supplémentaire selon laquelle chacun de ces espaces emporte avec lui un mode de temporalisation de l'histoire qui lui est spécifique, et l'idée qu'au bout du compte ce qui importe c'est l'analyse de l'articulation entre des blocs d'espace-temps dont les rythmes ou les vitesses sont foncièrement variables, toutes ces idées ont été largement accoutumées par l'historiographie à la suite des travaux de Fernand Braudel.

Dominique Pestre avait évoqué, pour sa part, le «le caractère décisif et l'efficace propre d'un ordonnancement précis des objets et des hommes dans l'espace pour la reproduction et la standardisation de nombreux résultats expérimentaux » (D. Pestre, 1995, p. 514) : sa remarque porte plus loin, selon moi, que sur les sciences expérimentales, et l'on peut généraliser son intuition. Il s'agirait de faire reconnaître la puissance formatrice de l'espace sur les activités de la science. La perspective ouverte est celle d'une géographie de la science, voire d'une géo-histoire des sciences. Ce qu'on propose ici, de façon tout à fait programmatique, c'est de mettre en œuvre cette approche spatialisante dans le domaine qui nous occupe (la culture scientifique) et sur le lieu même qui nous réunit (Rome), afin de faire apparaître la portée historiographique et épistémologique de cette approche.

On s'appuiera pour cela sur une double prise de conscience, du côté de l'historiographie d'une part, et du côté des théories sociales d'autre part. Concernant le premier aspect, on suivra volontiers les indications définies par Bernard Lepetit (B. Lepetit, 1986, 1999 ; voir aussi I. Laboulais, 2000) : l'espace n'est pas seulement un cadre, ou un support, des activités humaines, ni seulement, en outre, un objet de l'enquête historique, il est 
aussi une voie d'accès à la connaissance des faits du passé, un outil heuristique en tant que tel. L'historien définit l'espace qui lui permet de mettre en œuvre son analyse, et les questions centrales, alors, sont d'une part celle de l'échelle spatiale qui est choisie par l'historien pour développer son questionnement, et d'autre part celle des catégories spatiales qu'il mobilise. Le deuxième aspect qu'on pourrait évoquer sollicite plus directement la théorie sociale et les manières dont elle a envisagé le rôle de l'espace dans les activités humaines. David Livingstone a montré, à cet égard, toute l'importance des travaux d'Anthony Giddens, parmi d'autres, pour la reconnaissance et la compréhension des phénomènes de structuration spatiale (spatio-temporelle, pour être plus exact) de la vie sociale. L'espace ou plutôt les espaces dans lesquels se déploient les activités sociales (et la science est considérée de ce point de vue comme une activité sociale) possèdent un pouvoir de détermination sur la forme de ces activités et, partant, indirectement, sur leur contenu (il faudrait également évoquer l'impact des travaux de M. Foucault sur la géographie culturelle américaine ; cf. sur ce point F. Driver, 1994, et C. Philo, 1992). Il s'agirait alors de faire apparaître les morphologies et les dynamiques de ces espaces.

En tout état de cause, cette perspective « spatialisante » s'inscrit dans un déplacement général, auquel on assiste aujourd'hui, par rapport à ce qu'on pourrait appeler une histoire « platonicienne » des sciences, pour laquelle la considération des lieux et des espaces n'entrait pas de façon fondamentale dans l'analyse des conditions et des formes de la production des connaissances scientifiques. Il faut aller plus loin, surtout, que l'idée selon laquelle l'espace constitue un simple cadre ou un contexte de l'analyse. Il faut considérer l'espace comme une dimension déterminante dans la fabrication du savoir scientifique, et, surtout, comme une clé pour la compréhension des mécanismes de cette fabrication. En particulier, il faut s'intéresser plus précisément aux spatialités, matérielles et symboliques, qui sont mises en œuvre dans la 
production, la diffusion, et la réception des idées scientifiques, plus généralement dans l'activité scientifique considérée du point de vue social mais aussi logique et méthodologique.

Dans cette perspective, on explorera ici trois directions de travail, qui, insistons-y, sont tout à fait programmatiques. Et l'on prendra la géographie comme un champ d'investigation et d'observation, sur lequel les analyses vont pouvoir s'illustrer et s'articuler, même si l'on peut faire l'hypothèse, par ailleurs, que ces analyses ont une portée plus étendue. Trois thèmes donc :

- l'organisation des espaces du savoir géographique ;

- les parcours effectués concrètement par l'information géographique au sein de ces espaces ;

- les représentations spatiales ou plus exactement les schèmes spatiaux constitutifs du savoir géographique.

\section{I - L’organisation des espaces du savoir géographique}

La première direction de travail correspond à ce qui pourrait être appelé une analyse spatiale de la production et de la circulation du savoir et des représentations scientifiques, mais aussi des objets, des institutions et des instruments qui l'accompagnent. Il s'agit, dans cette perspective, d'introduire les concepts, les techniques d'analyse, les savoir-faire adoptés par les géographes contemporains et de les appliquer à l'étude des sciences (et en particulier de la géographie ${ }^{4}$ ).

Cette direction de travail met en œuvre simultanément une hypothèse théorique, une méthodologie, et un champ d'investigation.

a) L'hypothèse est que l'espace constitue un élément déterminant dans l'histoire des idées scientifiqques, tout comme il constitue un élément déterminant dans la plupart des activités sociales. Il ne s'agit pas de dire seulement que la production du savoir est localisée, 
ou encore qu'elle est simplement répartie dans des lieux qui sont les contextes effectifs de la production des idées et dont ces idées porteraient la marque. Il s'agit aussi et surtout de dire que l'espace « dans » lequel ce savoir est élaboré possède une vertu sui generis, une puissance structurante sur cette production même, bref qu'il y a une morphologie de l'histoire du savoir (de sa production et de sa circulation dans la culture). Les activités savantes, comme les autres activités sociales, tirent leur forme particulière des caractéristiques des espaces dans lesquels elles se développent (B. Lepetit, 1999, p. 139). Il s'agit, en d'autres termes, de faire apparaître, avec l'espace considéré dans sa spécificité, un autre niveau, un autre élément déterminant dans le processus de l'élaboration du savoir. Il y a un espace ou plutôt des espaces de la fabrication et de la diffusion du savoir, dont il s'agirait alors de définir la nature, les formes spécifiques, et les hiérarchies internes.

b) Ce qui conduit aux considérations de méthode. La géographie contemporaine a distingué en effet un certain nombre de concepts et de modèles méthodologiques, qu'elle met en œuvre dans l'analyse des phénomènes spatiaux de la vie sociale. La simple évocation de ces concepts possède à elle seule une portée programmatique et heuristique pour ce qui concerne l'histoire du savoir scientifique en tant que phénomène spatial à l'époque moderne.

La géographie a posé, par exemple, des questions relatives à la localisation des activités humaines et aux raisons d'être de ces localisations. Au-delà, elle a posé des questions relatives à la distance qui sépare les entités géographiques et aux relations (aux interactions spatiales) que ces entités entretiennent néanmoins dans cette distance même, à la nature et à la densité de ces relations. Le concept de réseau a permis de désigner de manière générale les lignes, les voies, les circuits conducteurs, etc., qui desservent une entité géographique et par l'intermédiaire desquelles cette entité se connecte à d'autres entités, et au-delà à des systèmes de circulation d'un rang supérieur. Points, lignes, surfaces: la géographie s'efforce par ailleurs d'identifier les configurations spatiales, les morphologies au sein desquelles les 
activités sociales se développent. Elle cherche à caractériser la structure interne de ces configurations, en mobilisant les concepts de discontinuité spatiale, de centre et de périphérie, qui lui permettent d'envisager les phénomènes de différenciation, et en particulier les phénomènes de polarité. Les concepts d'organisation, de hiérarchie, de système, ont permis en outre d'orienter la recherche géographique vers les formes de structuration des espaces, leur diversité et leur complexité. Un concept comme celui de diffusion spatiale, même s'il a fait l'objet de nombreuses discussions, a permis de structurer l'étude des processus mettant en jeu des déplacements de matière, de produits, de personnes, d'informations, d'idées, dans un milieu ou un territoire quelconques. On reviendra plus précisément dans un instant sur les concepts de niveaux de spatialisation et d'échelles spatiales, grâce auxquels il est devenu possible de penser des espaces de tailles différentes, c'est-à-dire d'envisager l'existence simultanée de phénomènes spatiaux se développant selon des ordres différents, et de se poser la question de l'articulation entre ces phénomènes d'échelles différentes.

c) Il serait bien difficile de décrire aujourd'hui avec précision les usages heuristiques qui pourraient être fait de cette batterie de concepts dans le domaine de l'histoire des sciences, et de la géographie en particulier. Nous sommes encore à cet égard dans un horizon programmatique. Certes, il serait possible de trouver dans des recherches plus anciennes sur la géographie de la première modernité des intuitions et aussi des matériaux qui pourraient être mobilisés en les reformulant au sein du programme indiqué ici. On pourrait, d'ailleurs, aller un peu plus loin sur ce point que la simple indication traditionnelle du déplacement des centres de la production géographique et cartographique en Europe, de l'Italie vers l'Europe du Nord, correspondant, en gros à ce que Fernand Braudel appelle le basculement, à partir du $\mathrm{XV}^{\mathrm{e}}$ siècle, des centres de l'économie-monde de la Méditerranée vers l'Europe du Nord. Il faudrait s'intéresser, également, aux circuits de diffusion de l'innovation scientifique en géographie (la diffusion des informations concernant les nouveaux mondes), aux relations 
centre/périphérie (Europe/reste du monde), aux situations de polarité et de marginalité dans la production du savoir (Anvers, Amsterdam, Venise, Rome, mais aussi Londres, Nuremberg, Mexico, etc.), aux routes (nationales et internationales) du savoir (les relations entre Italie et Pays-Bas, entre Italie et péninsule ibérique, la route du Rhin), aux politiques territoriales dans lesquelles la géographie en particulier est impliquée (détermination des voies commerciales, établissement des frontières, mise en valeur des terrains). Beaucoup d'éléments sont déjà disponibles, par l'intermédiaire des études qui ont été réalisées sur les écoles et/ou traditions nationales en géographie (J.-M. Besse, 2003). Reste néanmoins à effectuer le travail de « mise en espace » de cette période de l'histoire des sciences.

On pourrait solliciter, à titre d'exemple, les travaux de Steven Harris (S.J. Harris, 1998, 1999) à propos de la Compagnie de Jésus et surtout à propos de ce que l'on pourrait appeler l'espace d'acquisition et de traitement des connaissances géographiques que la Compagnie a mis en œuvre à la surface du globe dès ses premières années d'existence. La recherche d'Harris, dont il est possible de ne pas partager toutes les orientations cependant (en particulier l'utilisation du modèle de la corporation), met l'accent, à la suite des travaux de John Law (J. Law, 1986), sur la question de la maîtrise symbolique et pratique de la longue distance. Il s'intéresse à la manière dont un Ordre à vocation spirituelle et missionnaire, comme celui des Jésuites, mais aussi des entreprises commerciales comme la Compagnie des Indes orientales, ou des institutions d'origine politique comme le Conseil des Indes, mettent en place de façon systématique des dispositifs techniques et des réseaux de longue distance, grâce auxquels les informations géographiques peuvent être recueillies, transportées, traitées, synthétisées, puis remises en circulation dans la culture sous une forme standardisée, à partir des « centres de calcul» (B. Latour, 1989), que constituent ces institutions, plus exactement les bibliothèques et chambres des cartes de ces institutions ${ }^{5}$. Les analyses d'Harris mettent en évidence une question décisive: celle de l'échelle spatiale des pratiques scientifiques. 
Comparant les «métriques» respectives d'opérations telles que, d'une part, la construction d'un instrument d'observation ou la rédaction d'un traité, et d'autre part la cartographie de l'Amérique du Sud ou l'élaboration d'une taxinomie du vivant, Harris en conclut que des disciplines telles que la géographie, mais aussi l'histoire naturelle, l'hydrologie, ou la botanique médicale..., doivent être considérées comme des sciences « de grande échelle ${ }^{6}$, par le nombre des personnes et des institutions qu'elles mettent en œuvre ainsi que par la taille des réseaux et des distances qu'elles doivent contrôler. Alors que les disciplines scientifiques auxquelles on accorde traditionnellement, dans l'historiographie de la Révolution scientifique moderne, le premier rang, comme la physique ou les mathématiques, doivent être considérées de ce point de vue comme des sciences de petite échelle. On pourrait sans doute discuter ce jugement concernant la physique et les mathématiques. Mais il reste que l'approche spatiale conduit ici à une conséquence qui n'est pas négligeable : car elle permettrait de reconfigurer la représentation traditionnelle de la Révolution scientifique moderne, de réévaluer la notion de «science importante », et peut-être, souligne Harris, devrait-on réfléchir un peu plus profondément à la catégorie même de «révolution scientifique », lorsqu'on observe que les sciences sur lesquelles s'appuie ce modèle historiographique appartiennent toutes aux sciences de « petite échelle ».

On peut donc tenter de définir l'activité scientifique par l'intermédiaire des espaces de travail à l'intérieur desquels cette activité se déploie, et plus précisément par la taille de ces espaces de travail. En rappelant néanmoins un préalable : que le choix d'une échelle d'analyse spatiale, en géographie, détermine le choix du type de problème que l'on veut poser, du type d'objet que l'on veut analyser, et par conséquent du type de sources (et d'archives) que l'on veut interroger. Prenons l'exemple de la géographie jésuite, ce qui va nous rapprocher de Rome. Sans négliger la question des contenus spécifiques du savoir géographique des Jésuites, ainsi que celle des concepts et des théories qui structurent ce savoir, il est possible de 
chercher à faire apparaître, comme Harris a commencé à le faire, l'organisation de l'espace de la géographie des Jésuites. Plus précisément, la question qui est posée en ce cas est celle de la définition, de la maîtrise, et de l'entretien d'un espace de travail qui se développe à l'échelle du monde, à l'échelle du globe, c'est-à-dire à l'échelle des longues distances. Par quels moyens cet espace est-il établi ? Quels sont les canaux, ou les réseaux, qui le structurent ? Comment se déterminent (selon quelles règles et quels avatars ?) les relations entre le centre (les centres) et les périphéries de cet espace? Quelles sont les polarités de cet espace ? Quelles sont ses marges, et celles-ci sont-elles nettement définies ? On aperçoit tout de suite l'importance de ces questions pour déterminer le statut du « lieu-Rome » (ce lieu qui incarne exemplairement la superposition et la concentration des échelles) dans cet espace, ou bien pour mesurer la portée effective du système des relations épistolaires au sein de la Compagnie, ou bien encore pour évaluer le rôle joué par quelqu'un comme Kircher ainsi que la place du museum kircherianum au Collège romain dans l'économie globale de ce système spatial (P. Findlen, 1991, 1995).

Prenons un exemple, celui du fameux «horoscope jésuite», qui paraît être une expression très représentative de l'organisation de l'espace de travail de l'Ancienne Compagnie, et de son projet. Cet horoscope, placé par Kircher dans l'Ars magna lucis et umbrae ${ }^{7}$, est peut-être la plus élaborée des tentatives pour « cartographier » symboliquement l'espace d'influence structuré par la Compagnie. Sous la forme d'un arbre (analogue à l'arbre de Jessé) partant de Rome (et de ce centre dans le centre qu'est la méditation d'Ignace), sont distribuées les Assistances et Provinces dans lesquelles la Compagnie s'est installée. Unification de l'espace terrestre, parcouru en tous sens par les navires chargés de bonnes paroles, de bons livres, d'instructions, mais également d'informations savantes, et de marchandises. Unification des peuples aussi, sous le registre de l'esprit, où se décline en toutes les langues et dans toutes les directions (du levant au ponant) la même louange du nom 
de Dieu. Unification des temps, enfin et surtout : en chaque lieu de la surface de la Terre, il est possible, grâce à l'horoscope, de connaître l'heure de Rome et ainsi de s'y relier symboliquement. Cette image, qui conjugue les vertus de la carte, de l'organigramme, et du document programmatique, résume exemplairement la manière dont les Jésuites développent sur plusieurs plans conjointement leur projet universaliste. Mais il ne faut pas oublier que cet instrument est présenté d'abord dans la perspective d'un programme de recherche scientifique, celui de la détermination des longitudes. C'est dans le chapitre sur les longitudes que Kircher fait apparaître l'instrument, dans le cadre d'une argumentation qui met en valeur le rôle scientifique joué par les membres de la Compagnie dispersés aux quatre coins du monde. Tout se passe comme si, par cet instrument, Kircher répondait à la lettre envoyée à Naudé par Mersenne en 1639, dans laquelle ce dernier indiquait combien, grâce au réseau des Maisons et Collèges jésuites, il serait possible de procéder à des mesures permettant de connaître la variation magnétique sur toute la surface du globe, ainsi que les longitudes, « que l'on cherche depuis si longtemps » (Mersenne, Correspondance, VIII, 761). L'image de Kircher peut être considérée comme l'illustration parfaite d'un projet spatial se déployant simultanément sur les plans scientifiques, politiques, et spirituels.

Peut-être une comparaison serait-elle alors possible, dans cette perspective des « espaces de travail » de la géographie, avec Sébastien Münster, et plus généralement avec la cosmographie protestante des $\mathrm{XVI}^{\mathrm{e}}$ et $\mathrm{XVII}^{\mathrm{e}}$ siècles. L'étude de la correspondance liée à l'élaboration de la Cosmographia universalis montre en effet comment le cosmographe bâlois appuie son travail de description cosmographique sur un réseau principalement situé dans la partie septentrionale et orientale de l'Europe, et comment la structure et l'orientation de cet espace ont un impact déterminant sur l'image du monde qui est véhiculée dans l'ouvrage finalement édité. Les géographes, du moins jusqu'à un certain point, ne représentent pas exactement le même monde, ne donnent pas à penser le même monde, et cela est dû au réseau 
et à l'espace de travail qu'ils sont parvenus à mettre en place ou dans lequel ils sont parvenus à s'insérer, ou encore à leur aire d'influence.

Cette notion d'un espace de travail à l'intérieur duquel les géographes structurent leurs activités peut cependant être aussi déclinée à d'autres échelles. Jusqu'à présent, nous avons raisonné, à la suite de S.J. Harris, à l'échelle globale, et dans la perspective d'organisations humaines qui se donnent une vocation universelle. Mais on pourrait tout aussi bien porter l'attention, plus classiquement, sur la place de Rome à l'intérieur de l'espace européen de la production géographique, aux relations que Rome entretient avec les autres centres de production, en Italie (Venise, Gênes, Florence), et au-delà (Séville, Lisbonne, Anvers, Cologne, Paris). On peut envisager la production et la circulation des idées et des objets géographiques dans la perspective plus générale des espaces de l'échange marchand et savant qui se sont structurés en Europe à partir de la Renaissance. On pourrait ainsi dégager d'une part des réseaux européens (Pays-Bas/Italie, Italie/Péninsule ibérique, le monde des Habsbourg, l'aire hanséatique), d'autre part des discontinuités spatiales à l'intérieur de l'Europe (rôle des guerres, aires confessionnelles), qui jouent un rôle non négligeable dans l’organisation différenciée du savoir géographique.

À une autre échelle, locale celle-là, il est possible de s'arrêter aux localisations intraurbaines de la production géographique: où, dans la ville, élabore-t-on le savoir géographique ? Qui ? Quels groupes ? Quelles sociabilités ? Quel marché ? Local seulement? Ou jouant à d'autres échelles? Où trouve-t-on de la géographie dans la ville ? Ainsi à Rome la via del Pellegrino est devenue au cours du $\mathrm{XVI}^{\mathrm{e}}$ siècle le lieu où se rencontrent les antiquaires, les érudits romains, les graveurs, les amateurs d'estampes, pour acquérir les cartes, les plans, et les vues de la Rome antique et moderne, que l'on retrouvera ensuite dans différents lieux de la ville, collections ou bibliothèques. Et il serait possible d'étudier, à cette 
échelle, la répartition et la circulation des objets géographiques au sein de la ville et dans ses abords immédiats.

La question générale qui se pose alors est celle de l'articulation entre ces différentes échelles spatiales qu'on vient de distinguer : l'échelle de la cité et de ses environs, l'échelle intermédiaire de la nation et de l'Europe, l'échelle de la mappemonde (il faudrait affiner ces distinctions). Comment dans son activité le géographe parvient-il à parcourir ces différentes échelles, à les articuler, à les emboîter ? Deux hypothèses, pour conclure cette première série de remarques :

- Premièrement, il serait sans doute possible de dégager une corrélation (mais laquelle ?) entre, d'une part, la nature et l'échelle de l'espace de travail à l'intérieur duquel s'installe le géographe et, d'autre part, l'échelle spatiale qu'il cherche à représenter. On peut imaginer qu'il est nécessaire de disposer d'un réseau mondial ou en tout cas d'un réseau dont l'ampleur est extrêmement élargie pour pouvoir réaliser une mappemonde ou bien une carte du Nouveau Monde. Par contre il n'est peut-être pas nécessaire de disposer d'un tel réseau pour dessiner une vue topographique ou un plan de ville.

- Ce qui conduirait deuxièmement à une autre hypothèse : le degré de centralité d'un géographe se mesurerait à la possibilité qui serait la sienne de concentrer dans son travail plusieurs échelles, à sa possibilité de jouer simultanément de plusieurs niveaux de spatialité, c'est-à-dire aussi de jouer simultanément de plusieurs réseaux. Ainsi, la densité des relations spatiales, qu'elles soient sociales, savantes, ou spirituelles, dans lesquelles Kircher, pour revenir à lui, déploie son activité, serait le signe effectif de la centralité de sa position dans l'économie spatiale du savoir de son temps.

\section{II - Les trajets du savoir géographique}


La seconde direction de travail peut être considérée au départ comme un corollaire ou un prolongement des remarques précédentes. Rappelons ce que nous avons rencontré à propos de la science à l'échelle de la mappemonde. La géographie (tout comme l'histoire naturelle ou d'autres disciplines « descriptives ») peut être définie comme une science de grande échelle, dès lors que l'on tient compte, comme l'indique Harris, de la quantité des informations qu'elle doit traiter, du nombre de personnes qui y sont impliquées, de l'ampleur des espaces de travail qu'elle doit mettre en place, de la longueur et de la complexité des réseaux qu'elle doit contrôler. On ne revient pas sur cette caractérisation. Orientons maintenant l'attention sur un aspect, ou un enjeu, fondamental, de cette spatialité, qui est celui de la dynamique de l'élaboration de savoir, plus exactement celui des flux d'informations et des trajets effectués par l'information scientifique à l'intérieur de l'espace de travail de la géographie.

C'est, d'ailleurs, sans doute sur ce point, celui de la mobilité du savoir, que la distinction entre lieu et espace apparaîtrait de la façon la plus convaincante. On peut évoquer ici l'analyse développée par Michel de Certeau (M. de Certeau, 1980, pp. 208-209) : alors que le lieu est plutôt du côté de la « coexistence », de la « configuration instantanée de positions », de la stabilité et de l'inertie, il y a espace dès que des mouvements se déploient, dès que des mobilités, c'est-à-dire des temporalités, des orientations, des intentions, l'animent. L'espace, « est un lieu pratiqué », conclut-il. Mais, surtout, ces pratiques, ces opérations, qui transforment le lieu en espace, se caractérisent par le fait qu'elles permettent d'échapper à la « loi du propre » qui définit le lieu : l'espace signifie non seulement la mobilité mais aussi la transgression et la contamination, c'est-à-dire l'animation et la transformation du lieu par le « dehors » auquel il est confronté, ainsi que l'introduction d'une zone « d'étrangeté » au cœur même du lieu propre.

Il s'agirait alors, pour ce qui nous concerne, de s'interroger sur les relations matérielles pour ainsi dire «horizontales » à la surface du globe, sur les parcours concrets 
effectués par les données, scientifiques, géographiques, depuis les lieux où elles sont recueillies jusqu'aux lieux où elles sont traitées puis diffusées dans la culture. Bref comment, concrètement, l'information scientifique circule-t-elle ? Comment est-elle transportée depuis son lieu d'origine jusqu'à son lieu de représentation (et quelle est la nature de ce lieu de représentation)? Vers quels lieux est-elle destinée de façon préférentielle? Mais surtout comment, dans ce ou ces transports, ces déplacements, les données de la science se comportent-elles, si l'on peut dire ? Que deviennent-elles ? Par quels milieux passent-elles? Quelles transformations subissent-elles dans passages ? Dans quels conflits, éventuellement, sont-elles impliquées ? En un mot quel est leur devenir spatial?

Pour dire la chose autrement, l'information ne prend véritablement sa force objective que lorsqu'elle est mobilisée, c'est-à-dire à la fois rendue mobile, transportable et transposable hors de son lieu d'origine, et rendue utilisable pour la production de discours scientifiques nouveaux. Les données deviennent des faits lorsqu'elle sont connectées, justement, à des systèmes de transport et à des institutions de savoir, c'est-à-dire lorsqu'elles sont mises en mouvement à l'intérieur des réseaux de la science. La dynamique de l'activité scientifique (et de l'activité géographique au premier chef) consiste bien en ce cas, et quasi littéralement, en une mobilisation des mondes. Et l'on conçoit, à cet égard, toute l'importance stratégique qui s'attache d'une part à l'entretien des voies de communication et à la définition des règles de transport de l'information (la « métrologie »), et d'autre part à la détermination d'un ou plusieurs « centres » vers lesquels ces informations, c'est-à-dire ces «mondes » vont converger. Il semble que l'étude du mode de fonctionnement de grandes institutions telles que la Compagnie de Jésus, ou la Congrégation pour la Propagande de la Foi, pourrait constituer une illustration de cette hypothèse de recherche. Mais, outre les institutions, on peut également attirer l'attention vers des objets ou des espaces qui ont pour vertu de « récapituler 
le monde », pour ainsi dire : les atlas, les encyclopédies, les musées, constituent autant de « théâtres » au sein desquels le monde est conduit et concentré.

À condition cependant d'y ajouter une remarque, sans doute décisive. On pourrait la formuler ainsi : il n'y a pas de transport sans frottement, de déplacement d'information sans perte, bref la communication n'est pas synonyme de transparence de l'information depuis la source vers le lieu de destination. L'information se perd, au sens littéral du terme, elle s'égare, elle s'oublie, se trahit, ou tout simplement disparaît. Symétriquement, en même temps, il est possible d'indiquer en quoi l'information, durant son transport, est appropriée et réappropriée de multiples manières, et qu'elle est affectée, transformée par les milieux qu'elle traverse. L'information reçoit ou perd une signification à chaque moment, ou étape, du trajet qu'elle effectue.

Dans le cas précis de la géographie, on peut considérer que l'information est constamment délocalisée et relocalisée par le géographe, en fonction du niveau de signification spatiale qu'il lui accorde dans l'économie générale des concepts dont il dispose, mais aussi en fonction du type et de la complexité des supports ou lieux matériels à l'intérieur desquels cette information est accueillie et représentée (la narration, la carte, l'atlas, la collection d'objets) ${ }^{8}$. Allons plus loin : l'information géographique n'existe à vrai dire que dans cette série de figurations/traductions d'elle-même par laquelle elle traverse à la fois l'espace, le temps, et la culture. Plus exactement encore, elle n'existe que dans les multiples opérations de dé-localisation et re-localisation mentales et matérielles (graphiques le plus souvent) auxquelles elle est soumise et dont elle est le prétexte et le support.

Il s'agirait alors pour l'historien des sciences, comme on l'a indiqué, d'étudier le transport de l'information depuis son lieu d'origine jusqu'à son lieu de représentation (le livre, la carte, le musée, le jardin botanique), mais, surtout, il faudrait s'arrêter aux diverses opérations de reformulations ou de traductions que connait cette information pendant ce 
transport. Déclinons les questions : quels points de départ, quels points d'arrivée, quels relais, quels circuits conducteurs, quelles formes d'expression? Plus globalement, quel lien peut-on établir, ou conserver, entre l'information dans son lieu/contexte d'origine et l'information chargée de toutes les reformulations qu'elle a connues ? Quelles ruptures peut-on observer?

Deux exemples, de nature différentes. Le premier: la China illustrata de Kircher (1667). L'ouvrage est basé en grande partie, mais pas uniquement, sur les informations historiques et géographiques fournies à Kircher par des missionnaires jésuites : Benito de Goes, Heinrich Roth, John Grueber. Kircher, à la manière des cosmographes de son temps, compile et réécrit, à partir de leurs relations de voyage. Mais cette opération d'écriture prend en quelque sorte son indépendance vis-à-vis du matériel documentaire sur lequel elle s'appuie en partie. Ainsi, dans une lettre du 13 janvier 1670, John Grueber se plaint des nombreuses inexactitudes que contient le texte de Kircher. Illustration exemplaire d'un hiatus classique, si l'on peut dire, qui sépare les géographies de plein vent et les géographies de cabinet, où l'on voit les voyageurs ne pas se reconnaître dans les traductions de leur parole et de leur expérience.

Deuxième exemple, celui du trajet matériel et symbolique effectué par l'obélisque découvert en 1665 par les Dominicains de Santa Maria sopra Minerva lors du creusement des fondations d'un mur, puis érigé finalement, sur l'ordre d'Alexandre VII, au centre de la place sur laquelle se trouve l'église. La petitesse du trajet matériel ne fait peut-être que mieux ressortir, sur ce point, la vigueur du parcours symbolique effectué par cet obélisque. C'est-àdire la puissance de captation de l'objet qui se manifeste dans les opérations philologiques, artistiques, politiques, urbaines, suscitées par cet objet, et qui donnent lieu finalement, pour ainsi dire à plusieurs versions de cet objet. L'obélisque est pris à la fois dans un programme de connaissance (le livre de Kircher, Obelisci Aegyptiaci, 1666), dans un programme de type théologico-politique (les relations entre la papauté, l'Empire, et l'hermétisme), et plus 
directement dans un projet d'aménagement urbain qui est totalement investi de cette mission symbolique qui consiste à affirmer la centralité spatiale et temporelle de Rome (et l'on connaît l'importance, à cet égard, de la politique des obélisques dans les projets de structuration urbaine développés par la papauté).

Ces deux exemples nous signalent que le concept de mobilité spatiale n'est pas en luimême suffisant pour rendre compte de la situation épistémologique envisagée ici. Ce qui se transporte n'est pas une information immuable: car en réalité l'information bouge, se transforme, se reformule, est traduite selon les contextes qu'elle traverse et les situations où elle est réemployée. On pourrait dire, d'une certaine façon, que l'information traverse l'espace, chargée de ses différentes versions, et, qu'au fond, ce sont ces différentes versions d'elle-même, qu'elle connaît au cours des itinéraires qu'elle emprunte entre le site d'origine et le lieu d'arrivée, qui constituent son mode d'appartenance à la culture. Posons comme hypothèse que la culture scientifique d'une époque serait ce système herméneutique de traductions, de reformulations, ou de versions successives ou simultanées de l'information, versions qui lui donnent en fait sa véritable consistance, c'est-à-dire son épaisseur, au sein de la société. Un objectif de travail pourrait être de procéder à une cartographie de ce système.

\section{III - Les schèmes spatiaux mis en œuvre dans le savoir géographique}

On concentrera la troisième direction de travail, qui concerne l'étude de la spatialité du savoir scientifique, sur l'étude spécifique de ce qu'on nommera, peut-être un peu abusivement, une topique du savoir géographique. Plus exactement, on cherchera à envisager le savoir géographique du point de vue des représentations ou des schèmes spatiaux qui le sous-tendent.

Il ne suffit pas, en effet, pour rendre compte historiquement de la spatialité du savoir géographique, de dire que les géographes travaillent dans des lieux et des espaces qui 
définissent les conditions matérielles, sociales, culturelles, institutionnelles, de leurs activités (et l'on pourrait énumérer ici le terrain, le voyage, le cabinet, la chambre des cartes, le musée), ni de suivre les manières dont ces espaces sont parcourus et traversés par des flux d'information. Il faudrait sans doute ajouter que les géographes fabriquent pour ainsi dire des espaces mentaux qui viennent redoubler et accompagner les espaces matériels dont on a parlé jusqu'à présent. Ils élaborent leur conceptualité au sein d'espaces mentaux qui, eux-mêmes, sont aussi des espaces de travail, ces espaces s'inscrivant et se figurant en outre dans des objets, des représentations figuratives, ou des discours (schémas, graphiques, cartes, descriptions). La notion de topique du travail géographique, qu'on cherche à développer ici, correspond à une tentative pour reconstituer la structure de ces espaces mentaux, ou de ces schèmes d'espaces qui s'incarnent dans des dispositifs graphiques, plus précisément dans des espaces de représentation fabriqués par les géographes, et dans lesquels ces derniers inscrivent et développent leur pensée. C'est la nature et la logique propres de ces espaces destinés à produire et à représenter du savoir géographique, plus exactement ce sont les schèmes qui structurent ces espaces, qu'il s'agirait alors de faire apparaître.

Une remarque préalable à cet égard. Les historiens de l'imprimerie et les épistémologues ont montré à quel point, dans les procédures d'établissement des faits, les pratiques d'écriture et d'imagerie ont joué un rôle décisif. Ils ont voulu voir dans ces techniques d'inscription et d'enregistrement des opérateurs heuristiques, qui possèdent le pouvoir de produire des faits en organisant l'information pour le regard. Un grand nombre d'outils et d'auxiliaires de la recherche scientifique, qui ont pour caractéristique principale d'être des schématisations spatiales, se sont vus alors reconnaître une portée cognitive considérable : les cartes, diagrammes, listes, formules, archives, dossiers, dessins techniques, dictionnaires, collections de textes, ont été présentés comme autant de supports où les faits s'instituent et se révèlent (B. Latour, 1985). 
Si l'on reprend cette perspective, on peut reconnaître que l'élaboration du savoir géographique, au premier chef, se présente comme une entreprise qui n'est pas seulement de l'ordre de la mesure. La géographie est aussi un art du rangement des choses, et l'on peut espérer exhiber les schèmes sous-jacents à ces opérations de rangement. La géographie enveloppe des opérations d'enregistrement, de codification, de classement, d'archivage, de comparaison et de combinaison, bref tout un travail de type spatial sur les informations ellesmêmes, qui fait de la science non seulement une quête mais aussi une gestion spatiale des informations et de leurs inscriptions. Et il y aurait ainsi à mettre en place une authentique épistémologie du dossier et de l'archive, compris comme des moments constitutifs du fait géographique. Le fait géographique, en effet, est produit dans la collecte, le rassemblement, l'homogénéisation, l'accumulation des données. Il est lié à une procédure d'écriture et de gestion des signes, que ce soit dans la carte ou dans le texte descriptif, procédure panoptique dont il est le résultat et l'expression (M. Carpo, 1998). Le fait géographique se présente au sein d'un dispositif où s'ordonne la rencontre entre d'une part un « cumul documentaire » et d'autre part un «pouvoir d'écriture» (M. Foucault), qui est plus largement un pouvoir de représenter de façon homogène une grande diversité de documents. Il est le produit, et pour ainsi dire la cristallisation ou la stabilisation, de cette rencontre et de ce dispositif.

Il ne faudrait donc pas considérer les techniques d'inscription de l'information sur des supports seulement comme des instruments destinés à favoriser des stratégies promotionnelles en direction d'un public que l'on veut convaincre. Il faut y voir aussi des médiations essentielles dans les opérations proprement dites de construction des faits et des ordres d'objectivité. Il y a toute une dimension instituante de l'inscription, qu'il faut reconnaitre comme telle, et qui constitue une des clés d'analyse fondamentale pour une épistémologie historique de la géographie. La circulation des informations et des traces géographiques, leur accumulation, les multiples opérations de comparaison, combinaison, recouvrement, 
sélection, interprétation, auxquelles elles sont soumises, doivent être considérées comme autant de moments dans la constitution du savoir géographique (ce qu'on a appelé les différentes « versions du fait »). Le fait se présente comme le terme plus ou moins stable de cette série d'opérations. C'est la forme qui est issue de cette opération synoptique qui consiste à rassembler en un même lieu, et si possible de telle sorte que cela soit accessible pour un regard, des données extrêmement diverses.

La géographie de la première modernité, exploitant les modèles intellectuels offerts par Ptolémée et Strabon (« redécouverts » au cours du $\mathrm{XV}^{\mathrm{e}}$ siècle), offre un grand nombre de situations ou de réalisations exemplaires de cette opération synoptique. On peut les trouver dans l'histoire de la réception de la cartographie ptoléméenne, mais aussi dans l'histoire de l'élaboration de la formule descriptive dans les livres de cosmographie universelle (du type de celui de Münster, dans ses relations avec les arts de la mémoire, la rhétorique de l'éloge et la méthode des lieux communs), ou dans les premiers essais de mise au point de la formule de l'atlas (Ortelius, Lafrery, Mercator). À chaque fois, il s'agirait d'apercevoir la schématisation spatiale particulière que ces différents « objets » développent, aussi bien sur le plan des formes logiques qui y sont mises en œuvre que sur le plan matériel des modes d'inscription, ou des types de supports. Il faudrait travailler ici au ras des pratiques savantes, des techniques graphiques et des démarches de pensée, et chercher à faire apparaître les règles spatiales qui les conduisent et les organisent.

On peut ainsi repérer, aux $\mathrm{XVI}^{\mathrm{e}}$ et $\mathrm{XVII}{ }^{\mathrm{e}}$ siècles, au moins quatre schèmes spatiaux organisant l'élaboration du savoir géographique et sa présentation. Ces schèmes peuvent être juxtaposés, ou superposés, au sein d'une même œuvre. Mais à chaque fois, il faut comprendre que c'est une pensée spécifique de l'espace qui est engagée.

- Il y a d'abord le schème géométrique issu de Ptolémée (un Ptolémée, d'ailleurs, largement revisité et reformulé par les géographes de la Renaissance). Par l'intermédiaire des 
méthodes projectives et, surtout, de la méthode des coordonnées, c'est un espace « proportionné », « symétrique », « uniforme », qui se propose comme support de la pensée géographique et comme cadre de son travail.

- Mais les géographes trouvent aussi chez Ptolémée un autre vocabulaire, et surtout un autre principe de découpage et d'organisation de l'espace, qu'on pourrait appeler le schème des ordres de grandeur. Les réalités géographiques sont alors présentées et pensées en fonction de l'échelle spatiale dans laquelle on les considère, ces échelles successives pouvant d'ailleurs s'emboîter : cosmographie, géographie, chorographie, topographie, définissent à la fois un cadre de rassemblement des données et un style d'analyse de ces données pour le géographe.

- Un des schèmes spatiaux les plus volontiers utilisés par les géographes est le schème descriptif, dont la formule est progressivement mise au point au cours des $\mathrm{XV}^{\mathrm{e}}$ et $\mathrm{XVI}^{\mathrm{e}}$ siècles. Nous sommes là dans le voisinage des pensées de la collection, de l'encyclopédie, dans ce mouvement général de l'époque qui cherche à mettre le monde en fiches, pour ainsi dire. La géographie trouve des modèles opératoires, pour mettre en œuvre sa description, du côté des arts de la mémoire, de la méthode des lieux communs, ou de la rhétorique de l'éloge (J.-M. Besse, 2003). Dans cette configuration intellectuelle, la géographie à la fois cherche ses modèles ailleurs (la rhétorique) et offre un modèle pour d'autres pensées spatiales (l'encyclopédie).

- À la fin $\mathrm{du} \mathrm{XVI}^{\mathrm{e}}$ siècle, et surtout au XVII ${ }^{\mathrm{e}}$ siècle, se développe un nouveau schème spatial au sein de la géographie, le schème de la méthode, c'est-à-dire de la division dichotomique du général vers le particulier, que certains encyclopédistes protestants (Keckermann, Alsted) cherchent à faire coïncider, sans succès véritable, avec le principe ptoléméen des ordres de grandeur (J.-M. Besse, 2002). La division entre géographie et chorographie se reformule dans les termes d'une articulation hiérarchique entre géographie 
« générale » et géographie « spéciale ». On est là à la naissance de ce qui sera appelé ensuite la géographie générale (Varenius), et l'on pourrait, pour en revenir à la première partie de cet exposé, étudier de façon assez instructive les lieux d'élaboration de cette nouvelle pensée géographique, ainsi que les modes de sa diffusion (Amsterdam, en particulier). En tout état de cause, on peut observer la coexistence, au XVII ${ }^{\mathrm{e}}$ siècle, entre deux approches géographiques bien différentes, qui entretiennent d'ailleurs l'une et l'autre des relations différentes avec l'innovation copernicienne : une géographie générale d'une part (Varenius), et une géographie universelle d'autre part, qui prolonge la tradition descriptive.

La cartographie d'inspiration ptoléméenne, par sa puissance paradigmatique et prospective, va constituer un des schèmes fondamentaux permettant aux modernes de penser leur espace, mais aussi de le représenter et de le fabriquer réellement. Mais la géographie de l'époque moderne a mis en œuvre, comme on vient de l'indiquer, d'autres schèmes spatiaux lui permettant de donner une forme aux réalités terrestres qu'elle représente. On pourrait, tout aussi bien, suivre la manière dont les cartographes des $\mathrm{XVI}^{\mathrm{e}}$ et $\mathrm{XVII}{ }^{\mathrm{e}}$ siècles vont progressivement mettre au point la formule de l'atlas, en travaillant simultanément à différentes échelles et sur différents supports : le livre, la galerie, le studio, le jardin. Il serait très intéressant, à cet égard, de suivre les circulations, à l'intérieur de la ville de Rome ou bien depuis Rome vers les autres centres de production cartographique, entre le livre (Lafréry, Ortelius) et par exemple la galerie et le studio (Florence, Caprarola, Vatican). Dans ces circulations, ce qui se cherche et ce qui progressivement s'élabore, c'est la formule d'un espace de représentation, d'archivage, et de consultation, caractérisé par l'homogénéité des formats et des échelles, mais aussi par l'uniformité des symboles : cet espace a parfois été résumé dans le mot théâtre. 


\section{Conclusion}

Concluons en rappelant de façon synthétique les trois niveaux selon lesquels une approche spatiale peut être mise en œuvre au sein de l'histoire de la géographie, et plus largement de l'histoire des sciences.

Premier niveau: l'organisation spatiale du savoir scientifique. L'hypothèse développée ici est que cette organisation spatiale joue un rôle déterminant dans les processus d'acquisition, de formulation, et de diffusion du savoir. En bref, qu'il y a une configuration spatiale du savoir scientifique. Et la question serait alors celle de la situation de Rome dans cette configuration.

Deuxième niveau : les trajets spatiaux du savoir scientifique. Il s'agit ici d'analyser les multiples manières dont le savoir est mis en forme, mais aussi reformulé, et transformé, au cours des déplacements qu'il connaît. On a indiqué en quoi le savoir géographique s'incarne dans les versions successives auxquelles il donne lieu. On devrait souligner, alors, comment la notion d'une « culture géographique » correspond assez précisément à la co-présence de ces différentes versions du savoir géographique en un lieu et en un temps donné. Chacune de ces versions renvoyant pour ainsi dire aux autres, le système de ces renvois et de ces déplacements définit l'espace de la culture géographique. Une telle proposition est-elle applicable à d'autres secteurs, à d'autres régimes de savoir ?

Troisième niveau, plus spécifique: celui des schèmes spatiaux qui constituent l'armature des représentations géographiques. Nous sommes ici dans la perspective d'une psychologie ou d'une anthropologie historique, qui se préoccupe de restituer les logiques mentales, graphiques, et instrumentales, au sein desquelles s'élabore le savoir géographique. Là encore, on peut montrer comment Rome, notamment en ce qui concerne la mise au point de la formule de l'atlas, est un des lieux où se mettent en place et se développent ces schèmes 
spatiaux constitutifs du savoir géographique moderne. Là encore, la question se pose de la possibilité d'en dire autant pour d'autres types de savoir.

\section{Bibliographie :}

A. Bailly, R. Ferras, D. Pumain (dirs.), Encyclopédie de la géographie, Paris, 1992.

M. Beretta, Storia materiale della scienza. Dal libro ai laboratori, Milan, 2002.

J.-M. Besse, « L'analyse spatiale et le concept d'espace », dans J.-P. Auray, A. Bailly, P.-H. Derycke, J.-M. Huriot (éds.), Encyclopédie d'économie spatiale, Paris, 1994, pp. 3-11.

J.-M. Besse, « Le rôle de la carte dans la construction du concept de Terre aux quinzième et seizième siècles », Bulletin du Comité Français de cartographie, 103, 2000, pp. 6-15.

J.-M. Besse, « L'apparition de la notion de géographie générale en Europe au début du dixseptième siècle », texte issu d'un d'un exposé présenté le 18 mai 2002 au colloque « Structure et structuration de l'espace en Europe, du Moyen Âge à nos jours », organisé au Collegium Budapest par R. Albert, D. Iogna-Prat, D. Nordman, G. Sonkoly et A. Zempléni, à paraître. J.-M. Besse, Les Grandeurs de la Terre. Aspects du savoir géographique à la Renaissance, Lyon, 2003.

H. Bots et F. Waquet, La République des Lettres, Paris, 1997.

M.-N. Bourguet, C. Licoppe, H. Otto Sibum (eds.), Instruments, travels and science: itineraries of precision from the seventeeth to the twentieth-century, Londres, 2002.

N. Broc, La Géographie de la Renaissance (1420-1620), Paris, 1980.

M. Callon (éd.), La science et ses réseaux. Genèse et circulation des faits scientifiques, Paris, 1989. 
M. Carpo, «Descriptio urbis Romae: Ekfrasis geografica e cultura visuale all'alba della rivoluzione tipografica », Albertiana, vol. I, 1998, pp. 121-142. Texte repris dans L.B. Alberti, Descriptio Urbis Romae, traduction et commentaires M. Furno et M. Carpo, Genève, 2000.

M. de Certeau, L'écriture de l'histoire, Paris, 1975.

M. de Certeau, L'invention du quotidien. 1/ Arts de faire, Paris, 1980.

F. Dagognet, Le nombre et le lieu, Paris, 1984.

F. Driver, « Making Space », Ecumene, 1, 1994, pp. 386-390.

F. Driver, Geography militant. Cultures of exploration and Empire, Londres, 2001.

M.H. Edney, Mapping an Empire. The geographical construction of British India, Chicago \& Londres, 1997.

P. Findlen, « The economy of scientific exchange in early modern Italy », dans B.T. Moran (ed.), Science, Patronage, and Medecine at the European Court, 1500-1750, Rochester, 1991, pp. 5-24.

P. Findlen, «Scientific spectacle in baroque Rome. Athanasius Kircher and the Roman College Museum », Roma moderna e contemporanea, III, 3, 1995, pp. 625-665.

M. Foucault, « Des espaces autres », Dits et Ecrits, IV, Paris, 1994, pp. 752-761.

A. Giddens, La constitution de la société, Paris, 1984.

J.B. Harley, The new nature of maps. Essays in the history of cartography, edité par P. Laxton, Baltimore et Londres, 2000.

S.J. Harris, « Long-Distance Corporations, Big Sciences, and the Geography of Knowledge », Configurations, 6, 1998, pp. 269-304.

S.J. Harris, « Mapping Jesuit Science : The Role of Travel in the Geography of Science », dans J. O’Malley, S.J. Harris et G.A. Bayley (eds.), The Jesuits, Cultures, Sciences and the Arts, 1540-1773, Toronto, 1999, pp. 212-240. 
M.W. Helms, «Essay on objects: Interpretations of distance made tangible », dans S. B. Schwartz (ed.), Implicit Understandings. Observing, Reporting, and Reflecting on the Encounters between Europeans and other Peoples in the early Modern Era, Cambridge, 1994, pp. 355-377.

Laboulais-Lesage, «Les historiens français et les formes spatiales: questionnements et manières de faire (1986-1998) », dans J.-C. Waquet, O. Goerg et R. Rogers (éds.), Les espaces de l'historien, Strasbourg, 2000, pp. 32-50.

B. Latour, «Les 'vues' de l'esprit. Une introduction à l'anthropologie des sciences et des techniques », Culture technique, 14, 1985, pp. 5-29.

B. Latour, La science en action, Paris, 1989.

J. Law, « On the methods of long-distance control : vessels, navigation and the Portuguese route to India », dans J. Law (ed.), Power, Action and Belief : A New Sociology of Knowledge, Londres, 1986, pp. 234-263.

B. Lepetit, Carnet de croquis. Sur la connaissance historique, Paris, 1999.

B. Lepetit et P. Bourdelais, " Histoire et espace », dans espaces, jeux et enjeux, F. Auriac et R. Brunet (eds.), Paris, 1986, pp. 17-26.

D. N. Livingstone, « The spaces of knowledge : contributions towards a historical geography of science », Environment and Planning. D. Society and Space, 1995, pp. 13-42.

D.N. Livingstone, Putting science in its place. Geographies of scientific knowledge, Chicago, 2003

M. Lynch et K. Knorr-Cetina : The manufacture of knowledge. An essay on the constructivist and contextual nature of science, New York, 1981.

D. Outram, «New spaces in natural history », dans N. Jardine, J.A. Secord, E.C. Spary (ed), Cultures of natural history, Cambridge, 1996, pp. 249-265.

D. Parrochia, Philosophie des réseaux, Paris, 1993. 
D. Pestre, «Pour une histoire sociale et culturelle des sciences. Nouvelles définitions, nouveaux objets, nouvelles pratiques », Annales HSS, 3, 1995, pp. 487-522.

C. Philo, «Foucault's Geography », Environment and Planning D : Society and Space, vol. 10, 1992, pp. 137-161.

S. Shapin, «Here and Everywhere : Sociology of Scientific Knowledge », Annual Review of Sociology, 21, 1995, pp. 289-321.

A. Sophir et S. Shapin, «The Place of Knowledge. A Methodological Survey », Science in Context, 4, 1 (1991), pp. 3-21.

C.W.J. Withers, «Geography, natural history and the eighteenth-century enlightenment: putting the world in place », History Workshop Journal, 39, 1995, pp. 137-163.

1 Ce texte est issu d'un exposé présenté à Rome le 18 décembre 2000 lors de la Table ronde sur « Les lieux romains de la science », dans le cadre du groupe de recherche sur « La culture scientifique à Rome à l'époque moderne » (École Française de Rome/Centre Alexandre Koyré). Je remercie Antonella Romano pour ses remarques.

2 Je reprends cette expression à M. Dubois, Introduction à la sociologie des sciences, Paris, P.U.F., 1999, p. 215.

3 Voir encore récemment, A. Buttimer, S.D. Brunn et U. Wardenga (eds.), Social Construction of Regional Knowledges, Leipzig, Beiträge zur Regionalen Geographie, 49, 1999, ainsi que M.-C. Robic (dir.), Le Tableau de la géographie de la France de Paul Vidal de la Blache. Dans le labyrinthe des formes, Paris, CTHS, 2000.

$4 \quad$ Indiquons ici une fois pour toutes que les analyses qui suivent s'appuient avant tout sur le cas de la géographie. Cependant l'hypothèse est qu'elles peuvent prendre une portée plus générale. 
$5 \quad$ Les travaux de Felix Driver sur la géographie anglaise du $\mathrm{XIX}^{\mathrm{e}}$ siècle ont montré qu'il fallait utiliser le concept de «centre de calcul» avec prudence : ce concept en effet postule une homogénéité et une cohérence de la part des institutions de savoir, qui sont loin d'y correspondre dans leur réalité effective. Le savoir géographique est un domaine hétérogène, rappelle Driver, qui montre que la Royal Geographical Society est traversée par des courants et des intérêts très différents, entre lesquels les « négociations » sont constantes et les tensions persistantes. On appréciera, au passage, la remarque adressée par l'auteur à la notion de « centre de calcul»: «It is indeed difficult to characterize a body which finds room for missionaries, anti-slavery campaigners, roving explorers, mountaineers, antiquarians, geologists and naturalists under its umbrella as a coherent 'centre' at all » (F. Driver, 2001, p. 47).

6 Précisons que la notion d'échelle n'est pas prise ici dans son acception cartographique rigoureuse.

$7 \quad$ J'utilise l'édition d'Amsterdam, 1671.

8 Voir, pour cette question de la relocalisation, le travail exemplaire d'A. Pagden, European Encounters with the New World, New Haven \& London, Yale University Press, 1993. 